\title{
Ornella Vanoni, Ricetta di donna: alcune osservazioni linguistiche
}

\author{
Francesca Cialdini
}

PUBBLICATO: 31 DICEMBRE 2019

$R$ icetta di donna, interpretata da Ornella Vanoni, è un esempio di come un testo di una canzone, attraverso scelte linguistiche accurate, si soffermi con ironia su alcuni stereotipi che riguardano la donna ${ }^{\mathrm{I}}$. La canzone, contenuta nell'omonimo album Ricetta di donna (I980) ${ }^{2}$, è scritta dalla stessa cantante insieme a Amerigo Paolo Cassella, Sergio Bardotti, Totò Savio e Michele Zarrillo3.

Come noto, Ornella Vanoni è una delle più importanti cantanti italiane; alla fine degli anni Cinquanta, dopo l'esordio come attrice nella compagnia del Piccolo Teatro di Milano ${ }^{4}$, raggiunge i primi successi interpretando le canzoni della malavita milanese (la Mala)5. Tra gli anni Sessanta e Settanta fa parte del gruppo di cantanti e cantautori - tra i quali ricordiamo Jannacci, Endrigo, I Gufi - sicuramente diversi tra loro ma con in comune l'aspirazione alla scelta della realtà e di "interlocutori reali, per parlare di cose vere, in un modo che cerchi di farsi capire ${ }^{\prime \prime}$. L'esperienza cantautoriale di Ornella Vanoni negli anni Ottanta prosegue e nel r98 compone Vai Valentina7, rivolta a una giovane donna; negli anni Novanta la figura femminile viene tratteggiata nell'album Sherhazade, definito un "viaggio sentimentale nel mondo femminile", con canzoni scritte per la maggior parte dalla cantante stessa ${ }^{8}$.

In un arco cronologico compreso tra gli anni Settanta e oggi dai testi delle canzoni di Ornella Vanoni emergono diverse figure di donne, che fanno riferimento a mondi e a contesti socio-culturali anche lontani tra loro. Per esempio, in Vai Valentina viene rappresentata una giovane donna, Valentina, alla quale una donna più grande e più saggia rivolge alcuni consigli.

Le scelte linguistiche del testo sono interessanti e in particolare notiamo un'accuratezza nella selezione dei verbi, caratteristica che ricorre spesso nei testi di Vanoni. È senz'altro da rilevare l'uso dei verbi di movimento come andare (vai, presente anche nel titolo della canzone) e correre, che viene ripetuto più volte9 e introduce la similitudine corri come una gazzella che non vuol finire in mezzo ai trofei, critica all'immagine della donna vista come un trofeo; e ancora in Una bellissima ragazza (2007), di cui Vanoni è solo interprete, il lessico scelto pone l'accento sulla riflessione della donna: Come un entusiasmo antico / Mentre in mezzo a questa piazza / Sto ridendo a ripensarmi / Una bellissima ragazza ${ }^{\mathrm{IO}}$.

In Ricetta di donna l'attenzione è posta in modo ironico su alcune caratteristiche che dal punto di vista maschile una donna deve possedere: in molti casi si tratta di stereotipi che hanno origini lontane nel tempo. Come in una ricetta di cucina - che dal punto di vista testuale è un testo regolativo vengono utilizzati in incipit i verbi direttivi (deve avere), a indicare gli "ingredienti" e come utilizzarli: Deve avere l'aria di chi ha già vissuto / L'espressione fiera che non è un rifuto. Dunque, oltre all'esperienza, è richiesto un certo tipo di atteggiamento: la donna deve avere, infatti, un'espressione fiera.

Nei versi successivi vengono esplicitate le caratteristiche fisiche richieste a una donna: Seno quanto basta a riempir la mano / Fianchi dove affonda qualunque capitano. Interessante l'uso di capitano, che 
rinvia in modo diretto al potere attribuito all'uomo; inoltre il verbo affondare associato al sostantivo capitano rimanda all'immagine del comando di una nave.

La parte centrale del testo è occupata dal ritornello, in cui i verbi scelti hanno una forte connotazione semantica e danno un valore preciso a tutto il testo della canzone:

Costano le donne costano

Piu dei motori dei gioielli e delle lacrime

Ballano le donne ballano

Ma quelle vere sono rare

E non si comprano

Tu le puoi prendere ma non comprendere

I verbi costare e ballare sono ripetuti e posti allinizio di verso. In particolare, costare riproduce un luogo comune sulle donne: Costano le donne costano / Piu dei motori, dei gioielli e delle lacrime. Il concetto che lega le donne al denaro e alle cose materiali è antico: basti pensare alle molte espressioni proverbiali in cui il sostantivo donna/e ricorre insieme a denaro e ad altri nomi riferiti ai piaceri materiali. Ecco alcuni esempi tratti dalla banca dati sui Proverbi italiani ${ }^{\mathrm{II}}$ : "I danari fanno le donne puttane" che troviamo nella raccolta cinquecentesca di Francesco Serdonati; "Il molto donare fa le donne amare", presente oltre che in Serdonati anche nel Giardino di Ricreatione di John Florio ${ }^{\mathrm{I} 2}$; "Tre D rovinan l'uomo, Diavolo, Danaro e Donna" che ricorre nei Proverbi toscani di Giuseppe Giusti ${ }^{\mathrm{I}}$.

Espressioni formate dal sostantivo donna/e e il verbo costare ricorrono anche in letteratura, per esempio in Verga nel romanzo Tigre reale ( 1875 ) leggiamo: "Bassano avea fatto uneccellente speculazione sulla rendita lo stesso giorno, e tiro in campo il listino della Borsa a proposito di quanto costano le donne"I4.

In Ricetta di donna le donne costano più dei motori e dei gioielli: anche il sostantivo motori riferito a donna dà origine a proverbi noti come donne e motori, gioie e dolori. Questo proverbio ricorre anche in un'altra canzone italiana, Il poeta di Bruno Lauzi (I963), ma viene utilizzato con una funzione del tutto diversa. L'autore parla della sofferenza di un uomo a causa dell'amore per una donna e per descrivere la sensibilità dell'uomo (viene definito poeta) e la profondità di questo sentimento, in contrasto con i luoghi comuni utilizzati dagli altri uomini di un paese di provincia, utilizza queste parole: "Alla sera, al caffe, con gli amici / si parlava di donne e motori / si diceva "son gioie e dolori" / lui piangeva e parlava di te"

Nella canzone di Ornella Vanoni è presente anche un riferimento ai sentimenti, poiché le donne costano più delle lacrime, cioè possono essere più care della sofferenza.

Oltre a costare, l'altro verbo del ritornello è ballare: le donne ballano e in questo modo possono esprimere la propria femminilità. Ma non è possibile descrivere la realtà in base a categorie fisse e lo stereotipo viene messo in crisi: non a caso il verso inizia con la congiunzione avversativa ma. Vanoni non nega l'esistenza del tipo di donna appena descritto, ma focalizza l'attenzione sulle donne vere, che sono rare e non si comprano. Senza dubbio in questa frase l'uso dell'aggettivazione è importante: vere è utilizzato con il valore di 'reali' e 'schiette', ma anche di 'profonde, intense ${ }^{\mathrm{T} 6}$. 
Da notare, inoltre, nel ritornello l'opposizione verbale tra prendere e comprendere, legati dal punto di vista etimologico ${ }^{\mathrm{I}}$, ma distanti sul piano semantico. Prendere ha valore di 'sedurre'; comprendere di 'capire', con il prefisso con- che veicola senso di unione e di relazione ${ }^{\mathrm{I}}$. La differenza concettuale tra prendere e comprendere viene enfatizzata dall'uso del deittico tu, riferito all'uomo (Tu le puoi prendere ma non compredere).

La seconda strofa presenta l'immagine della donna vista come moglie:

$\begin{array}{ll}\text { Dice il talismano } & \text { E questa per un uomo } \\ \text { Che la buona moglie } & \text { Sarebbe lideale } \\ \text { Perde con il nome } & \text { Sarebbe lideale } \\ \text { Tutte le sue voglie } & \text { Ah ah ah ah } \\ \text { Stira lava e tace } & \text { Ah ah ah ah } \\ \text { Anche orizzontale } & \end{array}$

Nei primi versi troviamo anche una breve riflessione metalinguistica: il nome moglie concordato con l'aggettivo buona è distante semanticamente da donna (nel senso di 'donna non sposata') ${ }^{19}$; l'espressione buona moglie fa riferimento ai doveri della casalinga, che vengono elencati nel verso successivo attraverso una serie di verbi. Si tratta di un'espressione che troviamo, sempre in musica, ad esempio anche nell'opera buffa Olivo e Pasquale di Gaetano Donizetti ${ }^{20}$ : "Oliv. [...] A mia figlia darete la mano. / L'ho educata da vero Spartano: / Smorfie, vezzi, moïne non ha. / Non ha voglie -sarà buona moglie: / Tutto core, candore, onestà"2I.

Tuttavia, l'espressione buona moglie ricorre anche in proverbi dal valore decisamente più positivo, come risulta dalla consultazione del Tommaseo-Bellini, s.v. moglie: "Prov. Tosc. Ioz. La buona moglie fa il buon marito. [...] E 98. Chi incontra buona moglie ha gran fortuna. [...] Moglie buona, fa la casa; cattiva, la disfa"'22.

In Ricetta di donna ancora una volta i verbi stirare, lavare, tacere hanno un ruolo centrale: queste sono le funzioni richieste alla moglie. In particolare, sul fatto di tacere sono attestati proverbi antichi come «La donna dee parlare quando la gallina va a pisciare», già presente nella raccolta di Serdonati²3.

Dopo aver ricordato con ironia i "doveri" della donna, Ornella Vanoni si chiede (anche se non è presente il punto interrogativo deduciamo dal contesto e dall'intonazione il valore interrogativo della frase): "E questa per un uomo / Sarebbe l'ideale?". L'unica risposta possibile è ironica ed è rappresentata da una lunga e gustosa risata (Ah ah ah ah).

Nella terza strofa il punto di vista cambia: non si parla più della ricetta di donna secondo l'uomo, ma della ricetta di donna secondo la donna. Nella prima parte della canzone i verbi svolgono un ruolo importante, nell'ultima parte tutto ruota attorno agli aggettivi:

$\begin{array}{ll}\text { Bella non é tutto } & \text { Tenera e crudele } \\ \text { Meglio affascinante } & \text { Quando é innamorata } \\ \text { E una volta a letto dev'essere } & \text { Ma non avere miele } \\ \text { Importante } & \text { Per la fame di un pirata }\end{array}$


Secondo la cantante non basta essere bella, meglio essere affascinante (cioè 'che suscita interesse', 'che può incantare e attrarre anche dal punto di vista mentale ${ }^{24}$ ) e importante in riferimento al rapporto fisico con un uomo; attraverso un ossimoro la donna viene rappresentata come tenera e al tempo stesso crudele, se innamorata. La fine della strofa è introdotta dalla congiunzione avversativa ma ( $m a$ non avere miele per la fame di un pirata). L'uso di miele e pirata nella stessa frase mette ancora meglio in evidenza il consiglio rivolto alla donna: l'uomo, in quest'ultima parte, non è più definito capitano (come all'inizio), ma pirata, cioè senza una persona scrupoli.

Nella parte finale del testo, nell'ultimo ritornello (Costano le donne costano / Piú dei motori dei gioielli e delle lacrime / Ballano le donne ballano) è interessante l'uso dei verbi ritornare, piangere, rimpiangere. All'inizio del testo costare si opponeva a comprare (le donne vere non si comprano), in questo caso si oppone a ritornare preceduto dalla negazione:

La coppia verbale è formata da piangere e rimpiangere: quest'ultimo, usato nel senso di 'rivolgersi verso il passato con rammarico', deriva da piangere con il prefisso rin- (che indica un movimento inverso ${ }^{25}$. Come prendere e comprendere del primo ritornello, anche in questo caso i due verbi vengono utilizzati in opposizione.

Per concludere, la critica verso alcuni stereotipi femminili (anche antichi, risalenti al Cinquecento, abbiamo visto) avviene attraverso l'ironia, rappresentata senza dubbio dalla risata presente alla fine del ritornello, ma soprattutto linguisticamente attraverso l'uso degli aggettivi e in particolare dei verbi, presentati in coppia. Infatti è accurata la scelta di verbi come prendere/comprendere e piangere/rimpiangere, che mettono in evidenza anche la ricchezza della lingua: è possibile ottenere verbi, distanti dal punto di vista semantico, ma formati attraverso l'aggiunta di prefissi a una medesima base.

Le parole di questa canzone consentono di mettere a confronto "la ricetta di donna" dal punto di vista di un uomo con quella descritta da una donna e di evidenziare la difficoltà a fissare in categorie prestabilite il mondo femminile.

Note:

I. Il rapporto tra lingua e canzone è stato studiato nel corso del tempo e all'argomento è stato dedicato un Convegno ASLI (Associazione per la Storia della Lingua Italiana): Elisa Tonani (a cura di), Storia della lingua italiana e storia della musica. Atti del IV Convegno ASLI (Sanremo, 29-30 aprile 2004), Firenze, Cesati, 2005. All'interno del volume, per lo stato dell'arte fino a quel momento si veda il contributo di Lorenzo Coveri, Linguistica della canzone: lo stato dell'arte, pp. I79-9o. Successivamente in molti si sono occupati di questo argomento; per i principali riferimenti bibliografici sulla lingua della canzone rimando al contributo di Chiara Murru in questo numero.

2. L'album viene rilasciato dall'etichetta CGD e il produttore è Sergio Bardotti. Per ulteriori informazioni si veda il sito ufficiale di Ornella Vanoni: http://www.ornellavanoni.it/\#/dettaglio-disco.

3. Per la prima volta è coautrice, oltre di Ricetta di donna, di Amico mio, amore mio (Corrado Castellari - Ornella Vanoni - Cristiano Malgioglio - Sergio Bardotti).

4. Leonardo Colombati, Ornella Vanoni, in La canzone italiana 1861-2011. Storie e testi, Milano, Mondadori, 20II, pp. 865-77: 865. 
5. Enciclopedia Treccani, s.v. Ornella Vanoni: http://www.treccani.it/enciclopedia/ornella-vanoni/. Queste canzoni vengono scritte per lei in particolare da Giorgio Streheler, Dario Fo, Gino Negri e Fiorenzo Carpi.

6. Tullio De Mauro, Note sulla lingua dei cantautori dopo la rivoluzione degli anni Sessanta, in Lorenzo Coveri (a cura di), Parole in musica. Lingua e poesia nella canzone d'autore italiana. Saggi critici e antologia di testi di cantautori italiani, prefazione di Roberto Vecchioni, Novara, Interlinea edizioni, I996, pp. 3744: 4I.

7. La canzone è contenuta nell'album Duemilatrecentouno parole (Etichetta CGD; produttore Sergio Bardotti).

8. Leonardo Colombati, Ornella Vanoni, cit., p. 868.

9. Per esempio: "Corri, corri come un gatto / dal tuo letto alla fantasia / corri come la tua amica matta / dalla luna a una nuova bugia / E corri corri come corre il vento che se la gonna te la strappa una spina [...]; E allora corri corri come un ladro / che ha rubato un libro di poesie / corri corri che ti manca un metro / per salvare le tue unghie e le mie / e allora corri corri corri corri [...]".

Io. Sempre nello stesso testo: "Bisognerebbe tradurre sé stessi / Trovare un buon editore / Per poi tagliare un po' i discorsi / Che col tempo sono solo rumore".

II. La banca dati dell'Accademia della Crusca raccoglie un corpus che comprende le raccolte proverbiali cinquecentesche di Francesco Serdonati e Lionardo Salviati e quelle ottocentesche di Giusti e di Capponi. L'indirizzo è il seguente: http://www.proverbi-italiani.org/.

I2. John Florio, Giardino di Ricreatione nel quale crescono fronde, fiori e frutti, vaghe, leggiadri e soavi, sotto nome di sei mila proverbij, piacevoli riboboli italiani, colti e scelti da Giovanni Florio, non solo utili, ma dilettevoli per ogni spirito vado della nobil lingua italiana. Nuovamente posti in luce, Londra, appresso Thomaso Woodcock, I59I. Su questa raccolta proverbiale è basato in gran parte la Piazza Universale di Proverbi Italiani (I666) e i Select Proverbs (I642) di Giovanni Torriano: cfr. Herman W. Haller (a cura di), John Florio, A Worlde of Wordes. A critical edition with an introduction by Hermann W. Haller, Toronto-Buffalo-London, University of Toronto Press, 20I3, p. XXXVIII; Frances A. Yates, John Florio. The life of an Italian in Shakespeare's England,Cambridge, Cambridge University Press, 20II, p. 324. Su Torriano si veda da ultimo Lucilla Pizzoli, Giovanni Torriano e i Choyce Italian Dialogues (1657). Pratiche didattiche e modello di lingua usato da un maestro di italiano nell'Inghilterra del XVII secolo, in "Studi di grammatica italiana", XXXVII (20I8), pp. 95-II9.

I3. Giuseppe Giusti, Proverbi, a cura di Elisabetta Benucci, Firenze, Accademia della Crusca - Le Lettere, 20II, p. 2II.

I4. Fonte: BIZ - Biblioteca italiana Zanichelli, DVD-Rom per la ricerca in testi, biografie, trame e concordanze della letteratura italiana con il volume Biografie e trame; testi a cura di Pasquale Stoppelli, Bologna, Zanichelli, 20 оо.

I5. I corsivi sono miei. La citazione del testo della canzone è tratta dall'Antologia linguistica della canzone d'autore italiana, in L. Coveri (a cura di), Lingua e poesia, cit., p. r92. Negli anni Settanta il brano manifesto della scuola genovese era Il poeta di Bruno Lauzi (Giuseppe Antonelli, Ma cosa vuoi che sia una canzone. Mezzo secolo di italiano cantato, Bologna, il Mulino, 2007, p. I20 in nota). Per la lingua dei cantautori si veda da ultimo il volume di Vittorio Coletti, Lorenzo Coveri, Da San 
Francesco al rap: l'italiano in musica, Gruppo Editoriale L'Espresso, Accademia della Crusca - La Repubblica, 2016, pp. 74-85.

I6. Grande Dizionario Italiano dell'Uso, ideato e diretto da Tullio De Mauro, 6 voll., Torino, UTET, I999 (d'ora in avanti GRADIT), s.v. vero. Cfr. anche il Vocabolario Treccani, coordinamento scientifico di Valeria Della Valle, 5 voll., Roma, Istituto della Enciclopedia Italiana, 2008 (d'ora in avanti VOLIT), s.v. La scelta dei due aggettivi è precisa e il concetto di 'vero e raro' sembra essere importante per Ornella Vanoni, come lei stessa scrive nel libro Una bellissima ragazza. La mia vita: "Di uomini veri ce ne sono pochissimi, cosi come di donne. Le vere donne sono quelle che sostengono tutta quanta la baracca perché hanno la forza e il coraggio di farlo" (p. 234). Cfr. Ornella Vanoni, Giancarlo Dotto, Una bellissima ragazza. La mia vita, Milano, Mondadori, 201 .

I7. Comprendere deriva dalla forma latina comprehéndere, composta da cŭm + prehéndere: cfr. Dizionario Etimologico della Lingua Italiana, di Manlio Cortelazzo e Paolo Zolli, seconda edizione, a cura di Manlio Cortelazzo e Michele A. Cortelazzo, Bologna, Zanichelli, r999 (d'ora in avanti DELIN), s.v. comprendere.

I8. Claudio Iacobini, Prefissazione, in Maria Grossmann, Franz Rainer (a cura di), La formazione delle parole in italiano, Tübingen, Niemeyer, 2004, pp. 97-I63.

I9. In diacronia nella tradizione lessicografica italiana la definizione di donna come 'femmina dell'uomo' non è rara fino a tempi non troppo lontani (Paolo D'Achille, "Chi dice donna dice...". Le parole come strumento di infamia, in "Storia delle donne", 6/7 (2010/II), p. I3). La voce donna contenuta nelle cinque edizioni del Vocabolario degli Accademici della Crusca è interessante poiché le definizioni sono parzialmente differenti rispetto agli altri vocabolari. Nella prima edizione, s.v. donna gli Accademici riportano il significato "nome generico della femmina della spezie umana", senza il riferimento all'uomo, come invece fanno altri vocabolari. A partire dalla terza edizione del Vocabolario (I69I) compare in un sottolemma a sé il riferimento alla donna come moglie: "Donna: Moglie, maniera anche de' Greci. Boc. Nov. I6. 29. E rivoltosi alla sua donna disse: e a te, che ne parrebbe donna, se io cosi fatto genero ti donassi". Tra prima e quinta Crusca assistiamo a un notevole ampliamento della voce donna, grazie all'aggiunta di nuovi sottolemmi, nuove accezioni e anche modi di dire e proverbi; notiamo inoltre notiamo una certa continuità nei significati che tipicamente connotano la figura femminile: la donna viene vista come moglie, signora e padrona, madre e monaca. Sulla storia della parola donna nel Vocabolario della Crusca rimando a Nicoletta Maraschio, «Donna» e mestieri femminili: un piccolo sondaggio nelle cinque Crusche, in Paola Manni, Nicoletta Maraschio (a cura di), Da riva a riva. Studi di lingua e letteratura italiana per Ornella Castellani Pollidori, Firenze, Cesati, 20II, pp. 28595 .

20. L'opera viene rappresentata per la prima volta nel I827 e il libretto è di Jacopo Ferretti.

2I. I corsivi sono miei. Cito da Olivo e Pasquale. Melodramma giocoso. Musica di Gaetano Donizetti, Milano, per Antonio Fontana, i83o, p. I7.

22. I corsivi sono miei. Dizionario della lingua italiana, nuovamente compilato da Nicoló Tommaseo e Cav. Professore Bernardo Bellini [...], 4 voll., Torino, Unione tipografico-editrice, I86I-I879. Il dizionario è consultabile negli Scaffali digitali del sito dell'Accademia della Crusca all'indirizzo: http://www.tommaseobellini.it/. Cfr. anche il Grande dizionario della lingua italiana, a cura di Salvatore Battaglia [poi diretto da Giorgio Bàrberi Squarotti], 2I voll., Torino, UTET, I96I-2002. Con Supplemento 2004, diretto da Edoardo Sanguineti, Torino, UTET, 2004, e Indice degli autori citati nei 
volumi I-XXI e nel Supplemento 2004, a cura di Giovanni Ronco, Torino, UTET, 2004; e Supplemento 2009, diretto da Edoardo Sanguineti, Torino, UTET, 2008, s.v. moglie.

23. Più in generale, i proverbi con moglie sono numerosi e a parte il valore positivo in alcuni contesti di buona moglie, sono molti quelli negativi. Per esempio, nel Vocabolario degli Accademici della Crusca (I6I2), s.v. moglie: "[...] In proverbio per denotare che il duolo della moglie morta passa presto, si dice: Doglia di moglie morta / dura infino alla porta"; "Chi toglie una moglie merita una corona di pacienza, chi due, una di pazzia"; "Mal'anno, e moglie non manca mai". Si veda anche Helena Sanson, Donne, precettistica e lingua nell'Italia nel Cinquecento. Un contributo alla storia del pensiero linguistico, Firenze, Accademia della Crusca, 2007, in particolare alle pp. 62-76 sul "silenzio" della parola delle donne.

24. GRADIT e VOLIT, s.v. affascinante.

25. DELIN, s.V. rimpiangere.

\section{Cita come:}

Francesca Cialdini, Ornella Vanoni, Ricetta di donna: alcune osservazioni linguistiche , "Italiano digitale", 2019, XI, 2019/4 (ottobre-dicembre)

DOI: $10.35948 / 2532-9006 / 2020.3159$

Copyright 2019 Accademia della Crusca

Pubblicato con licenza creative commons CC BY-NC-ND 